\section{Preventing overuse injury in children}

- Careful supervision by coaches and parents

- Equipment checked regularly for fit and wear

- Practice intensity and duration increased only gradually

- Poor technique or posture recognised and corrected

- Warm up and stretch exercises before and after sport
Children who actively participate in sport should not be treated as miniature adults: they need careful assessment in relation to the common problems that affect a growing skeleton. Overuse syndromes can be avoided by careful supervision from coaches and sensible parents. General practitioners should refer children who are keen athletes to orthopaedic surgeons as soon as possible to allow early treatment and to avoid a long interruption of sporting activities.

Leslie Klenerman is professor of orthopaedic surgery at the University of Liverpool.

The ABC of Sports Medicine has been edited by Greg McLatchie, visiting professor of sports medicine and surgical sciences at the University of Sunderland, consultant surgeon at Hartlepool General Hospital, and director of the National Sports Medicine Institute, London. This paper has been edited in conjunction with Mark Harries, consultant physician at Northwick Park Hospital, Harrow, and director of clinical services at the British Olympic Medical Centre, Harrow.

\title{
Recent advances in tropical medicine
}

\author{
Diana N J Lockwood, Geoffrey Pasvol
}

Tropical medicine is perceived by many to be the preserve of pith helmeted colonialists who talk of obscure parasitic diseases. Reality in the late twentieth century is somewhat different, with post colonial clinicians, basic scientists, and epidemiologists contributing to our understanding of important public health problems. In this review we have chosen to focus on five tropical diseases (malaria, leprosy, leishmaniasis, onchocerciasis, and HIV) that exemplify recent advances in the molecular biology, immunology, and clinical practice of tropical medicine.

\section{Malaria}

A vaccine for malaria is still awaited. In 1993 the results were published of the SPf66 malarial vaccine developed and tested in Columbia by Manuel Patarroyo and his colleagues.' SPf66 is a synthetic peptide containing the amino acid sequences of three Plasmodium falciparum merozoite proteins linked to each other by a tetrapeptide derived from the circumsporozoite protein. In Columbia, an area of relatively low malaria endemicity, the vaccine had an overall protective efficacy of about $35 \%$, being highest in young children aged 1-4 years and adults over 45 years (at $77 \%$ and $67 \%$ respectively), but disappointingly only $22 \%$ in the 15-44 year age group. Three controlled trials, two in Africa (in the Gambia in children aged 6-12 months and in Tanzania in children aged 1-5 years) and one in Thailand (in children aged 2-15 years) are under way. Although safety and immunogenicity studies are promising, the efficacy of this vaccine in these areas of high transmission will not be known until August 1994. Development of other potential vaccines continues, and five further $P$ falciparum antigens from different stages of the life cycle have been earmarked by the World Health Organisation: three antigens involved in parasite invasion of red cells (merozoite surface protein-1 (MSP-1), apical membrane antigen (AMA-1), and erythrocyte binding antigen (EBA 175)); a soluble antigen (SERA) released when parasitised erythrocytes rupture; and-Pfs25, an antigen present on parasite zygotes.

The definitive diagnosis of malaria still relies on microscopic examination of thick and thin blood films. Recent attempts to develop other diagnostic techniques show how hard conventional methods are to beat. In the
QBC Malaria Test (Becton Dickinson), parasites are concentrated by centrifugation, stained with acridine orange, and visualised by fluorescent microscopy. This test is sensitive as well as labour and time saving, and large numbers of specimens can be processed. It is expensive, however, and cannot distinguish between malarial species. A polymerase chain reaction (PCR) based test has been developed and refined to a sensitive and specific, albeit cumbersome, method of diagnosis ${ }^{2}$; this has revealed a surprising number of unexpected mixed infections, particularly $P$ falciparum and $P$ vivax. The most promising recent development is a manual dipstick assay, the ParaSight-F test (Becton Dickinson), which detects a soluble antigen of $P$ falciparum, histidine rich protein-II (HRP-II). ${ }^{3}$ This rapid dipstick test requires no special equipment, and the result is read with the naked eye. Unfortunately, it will not replace tried and tested methods because it recognises only $P$ falciparum infections and is not quantitative. However, it can detect extremely low parasitaemias and may be useful in situations when treatment for falciparum malaria needs to be started without a blood film. In the field, the ParaSight-F test was found to be $99 \%$ sensitive and $94 \%$ specific, and when carried out daily during treatment it became negative later than both the thin blood film and the QBC methods. ${ }^{4}$ The ParaSight-F test has also been used to diagnose malaria in Egyptian and Nubian mummies, thus demonstrating the remarkable stability of HRP-II over several thousands of years. ${ }^{5}$

What can be regarded as new in the treatment of severe malaria when the drug quinine, whose use dates back to the 16th century, remains the mainstay of treatment? It is therefore remarkable that artemisinin, known and used in China for almost two thousand years was "rediscovered" only in 1971 when one of the active components, B-artemether was identified. Use of these compounds has been restricted to uncomplicated malaria, but now their value in cerebral malaria has been affirmed in several different studies. ${ }^{6-8}$ More widespread use of the artemisinin derivatives is appealing because of their unique pharmacodynamic action, 9 potential for shortening the period of coma in patients with cerebral malaria, and relat:-e lack of documented toxicity. Randomised controlled trials of artemether versus quinine in children with cerebral malaria are under way in the 


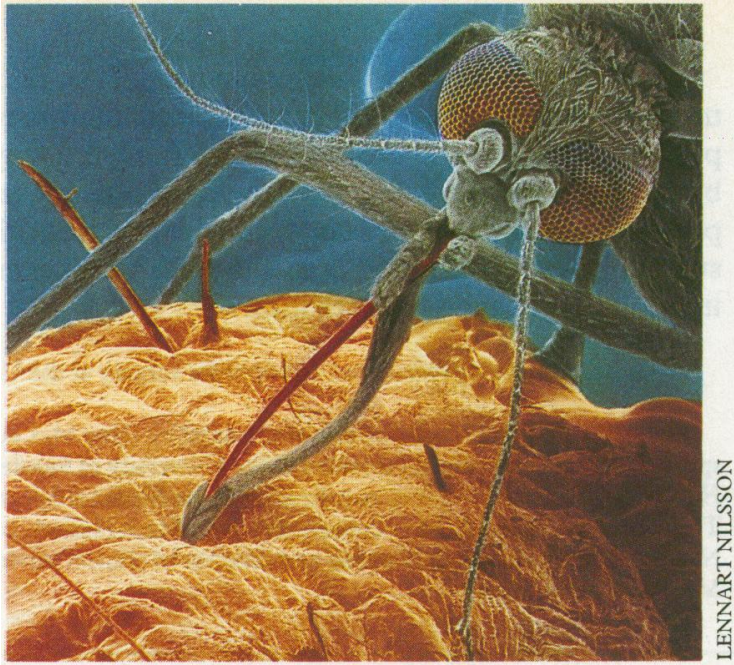

Mosquito drawing blood from a human arm

Gambia, Kenya, Malawi, and Nigeria; results are due later this year. Similar studies in adults in Thailand and Vietnam should be reported soon.

\section{Leprosy}

"Can leprosy be eradicated as a public health problem by the year 2000?" This was the goal set for leprologists by the WHO in May 1991. The WHO currently estimates that there are 4.4 million leprosy cases worldwide, a fall of about $50 \%$ since the 1980 s. This fall is in part due to the successful implementation of the WHO's recommendations for multidrug therapy, which has simplified leprosy drug treatment and which, with recommended treatment durations, has enabled many patients to stop taking antileprosy drugs. At the XIVth international leprosy congress in Orlando in 1993 there was much debate as to whether the WHO's goal is feasible and what the implications are for leprosy patients. A leprosy patient who has completed a course of multidrug therapy may be classified "cured" by the WHO, but many of these patients are left with severe disabilities requiring lifelong care and rehabilitation. The transformation of leprosy to a disease of low endemicity will surely have profound implications for the diagnosis of leprosy, delivery of treatment, and prevention of deformities as traditional leprosy clinics are integrated into general health services. ${ }^{10}$ The challenge will be to ensure that the particular needs of leprosy patients are still met.

Reversal reactions, episodes of immunological instability, are now recognised as being of major importance in the pathogenesis of leprous nerve damage. ${ }^{11}$ Borderline patients are at particular risk of this complication. ${ }^{12} 13$ Nerve damage results from intraneural granuloma formation and is exacerbated by the local expression of biologically important molecules such as TNF and heat shock proteins. ${ }^{14}$

Molecular biology has been spectacularly successful in leprosy with the development of a genetic map of Mycobacterium leprae DNA. Virtually complete coverage of the $M$ leprae chromosome has now been achieved and the position of 72 genetic loci determined. ${ }^{15}$ This work has produced one of the most comprehensive gene maps of any pathogen. $M$ leprae still cannot be cultivated in vitro so the production of individual recombinant $M$ leprae antigens is essential for studies of pathogenesis and drug resistance. Detection of rifampicin resistant $M$ leprae mutants with mouse footpad cultures currently takes $6-12$ months. Recently the mycobacterial gene encoding the target enzyme of rifampicin, the $\beta$ subunit of RNA polymerase, has been identified, ${ }^{16}$ and all currently known mutations producing rifampicin resistance have been shown to occur in a short segment of the gene (rpoB). By using a novel polymerase chain reaction amplification technique for examining the gene $\mathrm{rpoB}$, rifampicin resistant mutants may be detected within hours. ${ }^{17}$

Hopes that a single $M$ leprae protein or carbohydrate antigens might be associated with different patterns of immunopathology or protective immunity have not been realised. The outcome of infection with $M$ leprae is probably determined by the type of $\mathrm{T}$ cell that is activated. ${ }^{18}$ Tuberculoid disease results from a Th1type response in which T cells producing IL-2 and IFN- $\gamma$ predominate, whereas in lepromatous disease Th2-type cells predominate with production of IL-4, IL-5, and IL-10. ${ }^{19}$ It is not clear what factors drive T cells down $\mathrm{Th} 1$ or $\mathrm{Th} 2$ pathways.

Vaccine trials in Malawi and Venezuela have both shown that BCG gives substantial protection (vaccine efficacy $50 \%$ and $56 \%$ respectively) against leprosy. ${ }^{2021}$ Adding killed $M$ leprae to BCG does not seem to increase protection and at the moment there are no candidate $M$ leprae antigens for addition to BCG.

\section{Leishmaniasis}

Leishmaniasis is a dynamic disease, with new foci of disease being reported, such as visceral leishmaniasis, in Nicaragua and recognition of both insect and vertebrate vectors in new locations (Phlebotomus (Larroussius) orientalis in southern Sudan, and the gerbil in Saudi Arabia). New clinical manifestations of disease have also been described; American servicemen active in the Gulf war and infected there by Leishmania tropica have developed a chronic viscerotropic disease involving bone marrow and lymph nodes from this normally cutaneous parasite..$^{22}$

There are two major ongoing epidemics of visceral leishmaniasis (kala-azar), one in Bihar, India, and the other in the southern Sudan. ${ }^{23}$ The epidemic in Sudan is at least partly related to war and famine on the Ethiopia-Sudan border in the 1980s. Definitive diagnosis of leishmaniasis is demanding and time consuming, reliant on finding the parasite in bone marrow or splenic macrophages. Simple serological diagnostic tests would be useful in the rural conditions where epidemic disease occurs. A direct agglutination test, detecting antileishmanial agglutinating antibodies, has been successfully used in the field in both India and the Sudan. Direct agglutination testing was promising in India (sensitivity $96.5 \%$, specificity $100 \%),{ }^{24}$ but titres did not fall with recovery, making it a poor diagnostic tool in situations such as the Sudan where the prevalence of infection may be as high as $51 \%{ }^{25}$ An enzyme linked immunosorbent assay (ELISA) using soluble $L$ donovani protein antigens has recently been developed in Kenya and should make diagnosis of active cases in the field easier. ${ }^{26}$ Patients positive for HIV infection and coinfected with leishmaniasis pose a diagnostic problem because the leishmania serology may have negative results, but in this situation the recently developed $L$ donovani DNA probe, which can detect infections in aspirates and biopsy specimens, may be useful. ${ }^{27}$

Pentavalent antimonial agents given systemically for 30 days have been the mainstay of treatment for kala-azar since the 1940s. Unfortunately, one of the features of the current Indian epidemic is widespread therapeutic failure of antimony. An exciting prospect for treatment comes from the use of liposomal amphotericin B, which incorporates the drug into lipid vesicles which are then removed from the circulation by macrophages, the host cell ${ }^{\circ}$ for $L$ donovani. Higher total drug doses can be used, without appreciable toxicity. In a multicentre study 20 immunocompetent kala-azar patients treated with 
liposomal amphotericin B were cured and have not relapsed over a 12-24 month follow up period..$^{28}$

Kala-azar is now a common opportunistic infection in HIV infected patients who live in Mediterranean countries. Infection occurs particularly in periurban drug users and may be related to needle sharing. ${ }^{29}$ The parasite does not behave in a classic way in these immunosuppressed hosts, and disease may occur in unusual sites such as the gut, lungs, and central nervous system. Treatment is difficult, with relapse inevitable, even with liposomal amphotericin B treatment.

\section{Onchocerciasis}

Onchocerciasis (river blindness) is a filarial disease that is now being controlled and eliminated from large parts of West Africa through a WHO programme combining control of the blackfly vector and microfilarial eradication in infected humans. The programme, launched in 1974, uses aerial larvicides and has been estimated to have spared 9 million children from blindness without any long term effects on nontarget fauna. Since the adult female Onchocerca volvulus worm has an average life span of 11 years in its human host it was estimated that 14 years of spraying would be required to eliminate the adult worm reservoir in humans. The possibility of reinvasion by blackflies when spraying is discontinued, and the sheer expense of $\$ 20-30 \mathrm{~m}(£ 13 \cdot 3-20 \mathrm{~m})$ per year, are weaknesses of this particular approach. The timely discovery that ivermectin, a drug used in veterinary practice, is a highly effective microfilaricide has enabled a dual approach to be taken in onchocerciasis control. Unlike its predecessors ivermectin is safe, well tolerated, and effective in reducing microfilarial counts in both skin and eye. ${ }^{30}$ In 1993 a placebo controlled trial showed that villagers given ivermectin had a reduced incidence of optic nerve disease. ${ }^{31}$ Thus annual delivery of ivermectin to residents in endemic communities could prevent a substantial proportion of onchocercal blindness.

The success of ivermectin treatment in onchocercal infection has given further impetus to the development of serological tests. Previously, infection levels were monitored by the numbers of microfilaria found in skin snips, but this test is insensitive when ivermectin has reduced the microfilarial burden. Skin snip testing also fails to detect prepatent infections. Current serological tests based on parasite extracts are unsatisfactory because there is extensive cross reaction between parasite nematodes. A WHO multicentre trial ranking the specificity and sensitivity of available recombinant antigens failed to find a single antigen that was recognised by serum from all onchocercal patients. ${ }^{32}$ Bradley has used a cocktail of three recombinant proteins and achieved a test with $96 \%$ sensitivity and $100 \%$ specificity. ${ }^{33}$ This should prove useful for seroepidemiological studies detecting prepatent infection in control areas. The IgG subclass response to the recombinant antigen cocktail showed that the IgG1 response declined after interruption of transmission but the IgG4 response remained raised even eight years after transmission had stopped. ${ }^{34}$ An assay based on the polymerase chain reaction has also been developed that uses primers and probes specific to Onchocerca volvulus and has achieved a sensitivity of $100 \% .^{35}$

IgG antibodies play a major role in protective immunity to $O$ volvulus, with infected individuals having paradoxically high IgG4 levels and individuals with putative protective immunity having significantly lower worm specific antibodies than infected subjects. ${ }^{36}{ }^{37}$ Increased IgG4 levels were correlated with clinical severity and microfilarial load. IgG4 may be induced by worm or microfilarial antigens and may contribute to the immunopathology of disease by blocking microfilarial clearance and destruction.

\section{HIV in the tropics}

HIV infection, though not a classical tropical disease, is having a profound effect on the practice of medicine in the tropics. Fortunately there is little evidence for specific interactions between HIV infection and the major tropical diseases with the exception of leishmaniasis. However, HIV immunosuppression influences the responses that an individual can mount to common respiratory and enteric pathogens, and a distinctive clinical pattern of HIV disease in the tropics is emerging. ${ }^{38}$

Early HIV disease is associated with typical pulmonary tuberculosis and pneumococcal pneumonia, whereas later HIV disease is characterised by atypical diseases such as invasive salmonella and staphylococcal infections and extrapulmonary tuberculosis. ${ }^{39}$ The opportunistic infections seen in industrialised countries are rare because patients often die soon after their first major HIV disease. ${ }^{40} \mathrm{~A}$ strong association has emerged between HIV infection and tuberculosis with 20-67\% of tuberculosis cases in sub-Saharan Africa attributable to HIV infection. ${ }^{41}$ A comprehensive postmortem study in the Ivory Coast showed tuberculosis to be the cause of death in $30 \%$ and present in $54 \%$ of those dying with an AIDS defining pathology. Pneumocystis carinii pneumonia was present in only $4 \%$ of HIV cadavers. ${ }^{42}$

Is tuberculosis in African HIV patients due to reactivation or reinfection? A small study from Nairobi that used genetic fingerprinting of Mycobacterium tuberculosis isolates suggests that both occur: ${ }^{43}$ Larger studies are being done in Lusaka and Nairobi to answer this question. Whether HIV infected patients should receive chemoprophylaxis poses an urgent question for research. ${ }^{44}$ The resurgence of tuberculosis in association with HIV has implications not only for the individuals with disease but also for their community.

\section{Conclusion}

The complex relations between parasites, vectors, populations, and environments attest to the fact that tropical medicine is in a state of constant evolution. The challenge of these diseases in the modern world lies in applying up to date biotechnology to the understanding of these diseases, developing new diagnostic methods and drugs, and delivering appropriate patient care.

We thank Dr R N Davidson for his helpful discussions and constructive, critical reading of this review.

1 Valero MV, Amador LR, Galindo C, Figueroa J, Bello MS, Murillo LA Vaccination with SPf66, a chemically synthesised vaccine, against Plasmodium falciparum malaria in Colombia. Lancet 1993;341:705-10.

2 Snounou G, Viriyakosol S, Zhu XP, Jarra W, Pinheiro L, Rosario VE. Identification of the four human malarial parasites by the use of the nested
Intiounou polymerase chain reaction. Mol Biochem Parasitol 1993;58:283-92.

3 Swift CJ, Premji Z, Minjas JN. The rapid manual ParaSight-F test. A new diagnostic tool for Plasmodium falciparum infection. Trans Roy Soc Trop Med Hyg 1993;87:646-8.

4 Beadle C, Long GW, Weiss WR, McElroy PD, Maret SM, Oloo AJ, et al Diagnosis of malaria by detection of Plasmodium falciparum HRP-2 antigen with a rapid dipstick antigen-capture assay. Lancet 1994;343:564-8.

5 Miller RL, Ikram S, Armelagos GJ, Walker R, Harer WB, Shiff CJ, et al. Diagnosis of Plasmodium falciparum infections in mummies using the rapid manual ParaSight-F test. Trans Roy Soc Trop Med Hyg 1994;88:31-2.

6 Win K, Than M, Thwe Y. Comparison of combinations of parenteral artemisinin derivatives plus oral mefloquine with intravenous quinine plus artemisinin derivatives plus oral mefloquine with intravenous quinine p
oral tetracycline for treating cerebral malaria. Bull WHO 1992;70:777-82.

7 White NJ, Waller D, Crawley J, Nosten F, Chapman D, Brewster D, et al Comparison of artemether and chloroquine for severe malaria in Gambian children. Lancet 1994;339:317-21.

8 Taylor TE, Wills BA, Kazembe P, Chisale M, Wirima JJ, Ratsma EYEC, et al. Rapid coma resolution with artemether in Malawian children with cerebral malaria. Lancet 1994;341:661-2.

9 Ter Kuile F, White NJ, Holloway P, Pasvol G, Krishna S. Plasmodium falciparum; in vitro studies of the pharmacodynamic properties of drugs used for the treatment of severe malaria. Exp Parasitol 1994;76:85-95. 
10 Feenstra $P$. Leprosy control through general health services and/or combined programmes. Lepr Rev 1993;64:89-96.

11 Lienhardt C, Fine PEM. Type 1 reaction, neuritis and disability in leprosy. What is the current epidemiological situation? Lepr Rev 1994;65:9-33.

12 Lockwood DNJ, Vinayakumar S, Stanley JNA, McAdam KPWJ, Colston MJ. Clinical features and outcome of reversal (type 1) reactions in Hyderabad, India. Int 9 Lepr 1993;60:8-15.

13 Becx-Bleumink $M$, Berhe $D$. Occurrence of reactions, their diagnosis and management in leprosy patients treated with multidrug therapy: experience in the leprosy control program of the All Africa Leprosy and Rehabilitation in the leprosy control program of the All Africa Leprosy and Reh

14 Khanolkar-Young S, Young DB, Colston MJ, Stanley JNA, Lockwood DNJ. Nerve and skin damage in leprosy is associated with increased intra-lesional heat shock protein. Clin Exp Immunol 1994;96:208-13.

15 Eiglmeier K, Honore N, Woods SA, Caudron B, Cole ST. Use of an ordered cosmid library to deduce the genomic organisation of Mycobacterium leprae. Mol Microbiol 1993;7:197-206.

16 Telenti A, Imboden P, Marchesi F, Lowrie D, Cole ST, Colston MJ, et al. Detection of rifampicin-resistance mutations in Mycobacterium tuberculosis. Lancet 1993;341:647-50.

17 Honore N, Cole ST. The molecular basis of rifampicin-resistance in Mycobacterium leprae. Antimicrobial Agents and Chemotherapy 1993;37: $414-8$.

18 Britton WJ. Immunology of leprosy. Trans Roy Soc Trop Med Hyg 1993;87: 508-14.

19 Yamamura $M$, Uyemura K, Deans RJ, Weinberg K, Rea TH, Bloom TBR, et al. Defining protective responses to pathogens: cytokine profiles in leprosy lesions. Science 1991;254:277-9.

20 Ponnighaus JM, Fine PEM, Steme JAC, Wilson RJ, Msosa E, Gruer PJK, et al. Efficacy of BCG vaccine against leprosy and tuberculosis in northern Malawi. Lancet 1992;339:636-9.

21 Convit J, Sampson C, Zuniga M, Smith PG, Plata J, Silva J, et al. Immunoprophylactic trial with combined Mycobacterium leprae/BCG vaccine against leprosy: preliminary results. Lancet 1992;339:446-50.

22 Magill A, Grogl M, Gasser RA, Sun W, Oster CN. Visceral infection caused by Leishmania tropica in veterans of operation Desert Storm. $N$ Engl $\mathcal{f}$ Med 1993;328:1383-7.

23 Lockwood DNJ. Sudan: kala-azar should be in the news. Lancet 1991;338: 624-5.

24 Singla N, Singh GS, Sundar S, Vinayak VK. Evaluation of the direct agglutination test as an immunodiagnostic tool for kala-azar in India. Trans Roy Soc Trop Med Hyg 1993;87:276-8.

25 Zilistra EE, Siddig Ali M, El-Hassan AM, El-Toum IA, Satti M, Ghalib HW, et al. Kala-azar: a comparative study of parasitological methods and the direct agglutination test in diagnosis. Trans Roy Soc Trop Med Hyg 1992;86:505-7.

26 Okong'o-Odera EA, Wamachi A, Kagai JM, Kurtzhals JAL, Githure II, Hey AS, et al. Field application of an ELISA using redefined Leishmania antigens for the detection of visceral leishmaniasis. Trans Roy Soc Trop Med Hyg 1993;87:423-4.
27 Howard MK, Ogunkolade W, Bryceson ADM, Davidson RN, Moody AH, Miles MA. A DNA probe for human visceral leishmaniasis. Trans Roy Soc Trop Med Hyg 1992;86:35-6.

28 Davidson RN, Di Martino L, Gradoni L, Giacchino R, Russo R, Gaeta GB, et al. Liposomalamphotericin $B$ (Ambisome) in Mediterranean visceral leishmaniasis: a multicentre trial. $Q$ f Med 1994:87:75-81.

29 Alvar J. Leishmaniasis and AIDS co-infection: the Spanish example. Parasitology Today 1994;10:160-3.

30 Mabey DM. Ivermectin and onchocercal optic nerve lesions. Lancet 1993;341: 153-4.

31 Abiose A, Jones BR, Cousens SN, Murdoch I, Cassels-Brown A, Babalola OE, et al. Reduction in incidence of optic nerve disease with annual ivermectin to control onchocerciasis. Lancet 1993;341:130-4.

32 Ramachandran CP. Improved immunodiagnostic tests to monitor onchocerciasis control programmes-a multicenter effort. Parasitology Today 1993;9:76-9.

33 Bradley JE, Trenholme KR, Gillespie AJ, Guderian R, Titanji V, Hong Y, et al. A sensitive serodiagnostic test for onchocerciasis using a cocktail of recombinant antigens. Am f Trop Med Hyg 1993;48:198-204.

34 Bradley JE, Gillespie AJ, Trenholme KR, Karam M. The effects of vector control on the antibody response to antigens of Onchocerca volvulus. Parasitology 1993;106:363-70.

35 Zimmerman PA, Guderian RH, Araujo E. Polymerase chain reaction-based diagnosis of Onchocerca volvulus infection:improved detection of patients with onchocerciasis. FInfect Dis 1994;169:686-9.

36 Dafa'Alla YH, Ghalib HW, Abdelmageed A, Williams JF. The profile of IgG and IgG subclasses of onchocerciasis patients. Clin Exp Immunol 1992;88: 258-63.

37 Elson LH, Guderian RH, Araujo E, Bradley JE, Days A, Nutman TB. Immunity to onchocerciasis: identification of a putatively immune population in a hyperendemic area of Ecuador. F Infect Dis 1994;169:588-94.

38 Gilks CF. The clinical challenge of the HIV epidemic in the developing world. Lancet 1993;342:1037-9.

39 Gilks CF, Otieno LS, Brindle RJ, Newnham RS, Lule GN, Were JBO, et al. The presentation and outcome of HIV-related disease in Nairobi. $Q f \mathrm{Med}$ 1992;82:25-32.

40 Nunn P, Brindle RJ, Carpenter L, Odhiambo J, Wasunna K, Newnham RS, et al. Cohort study of human immunodeficiency virus infection in patients with tuberculosis in Nairobi, Kenya. Am Rev Respir Dis 1992;146: patients

41 De Cock KM, Coulibaly IM, Lucas SB. Tuberculosis and HIV infection in sub-Saharan Africa. ҰAMA 1992;268:1581-7.

42 Lucas SB, Hounnou A, Pecock C, Beaumel A, Djomand G, N'Gbich JM, et al. The mortality and pathology of HIV infection in a West African city. AIDS 1993;7:1569-79.

43 Hawken $M$, Nunn $P$, Gathua $S$, Brindle R, Godfrey-Fausett P, Githui W, et al. Increased recurrence of tuberculosis in HIV-1-infected patients in Kenya. Lancet 1993;342:332-7.

44 De Cock K. Impact of interaction with HIV. In: Porter JDH, McAdam KPWJ, eds. Tuberculosis: back to the future. Chicester: Wiley, 1994;35-49.

\title{
Lesson of the Week
}

\section{Upper abdominal pain in pregnancy may indicate pre-eclampsia}

\author{
Christopher Barry, Robert Fox, Gordon Stirrat
}

Clinical and laboratory tests for pre-eclampsia must be undertaken in all women with upper abdominal pain in pregnancy
University Department of

Obstetrics and

Gynaecology, St Michael's

Hospital, Bristol BS2 8EG

Christopher Barry, obstetric registrar

Robert Fox, lecturer senior

registrar

Gordon Stirrat, professor

Correspondence to:

DrFox.

$B M \mathcal{F} 1994 ; 308: 1562-3$
Pre-eclampsia is one of the prime causes of maternal and perinatal morbidity and mortality. The syndrome is highly variable, and the clinical presentation is often atypical. We describe the case histories of three women who presented with epigastric pain as the first outward manifestation of the disorder and in whom the diagnosis was initially overlooked.

\section{Case reports}

CASE 1

A 28 year old primigravida attended her general practitioner's surgery at 34 weeks' gestation with pain in the right upper quadrant, nausea, and vomiting. An antacid was prescribed for presumed reflux oesophagitis. Her blood pressure was not measured and her urine was not tested for protein. The symptoms persisted, and five days later she presented to the hospital antenatal clinic. On examination her blood pressure was $140 / 80 \mathrm{~mm} \mathrm{Hg}$ and urine analysis showed proteinuria $(2+)$. She had appreciable tenderness of the right hypochondrium. Further investigation disclosed a moderate thrombocytopenia $\left(56 \times 10^{9} / 1\right)$ and raised serum concentrations of aspartate aminotransferase $(225 \mathrm{U} / \mathrm{l})$ and uric acid $(0.37 \mathrm{mmol} / \mathrm{l})$. Concentrations of haemoglobin, urea, alkaline phosphatase, and albumin all lay within the normal ranges for pregnancy. There was no evidence of haemolysis, and the standard clotting times were not prolonged. A midstream urine sample was clear of white and red cells at microscopy. Pre-eclampsia with raised liver enzyme concentrations and a low platelet count was diagnosed. As the cervix was unfavourable caesarean section was performed to deliver the baby, a girl weighing $2100 \mathrm{~g}$; she required minimal resuscitation. With intensive monitoring and supportive care, the woman made a complete recovery over 72 hours, her blood pressure, aspartate aminotransferase concentration, and platelet count all returning to within normal limits.

\section{CASE 2}

A 26 year old woman complained of epigastric pain at 36 weeks in her first pregnancy. Her general practitioner diagnosed dyspepsia and prescribed an antacid mixture. Her blood pressure and urine were not checked. The pain continued, and at a routine antenatal visit two days later she was found to be hypertensive $(135 / 105 \mathrm{~mm} \mathrm{Hg})$ and to have proteinuria $(2+)$. On investigation in hospital that day she had moderate thrombocytopenia $\left(78 \times 10^{9} / 1\right)$ and raised serum concentrations of aspartate aminotransferase $(142 \mathrm{U} / \mathrm{l})$ and uric acid $(0.41 \mathrm{mmol} / \mathrm{l})$. Standard clotting times were normal and there was no evidence of haemolysis. The fetus was normally grown and had a reactive heart rate pattern. An attempt was made to induce labour with dinoprostone gel $(2 \mathrm{mg}$, two doses, 